\title{
Anabases
}

ANABASES Traditions et réceptions de l'Antiquité

18 | 2013

Varia

\section{Les thrillers de Daniel Chavarría, ou la rencontre de l'érudition classique et du polar cubain}

\section{Magali Soulatges}

\section{(2) OpenEdition}

1 Journals

Édition électronique

URL : http://journals.openedition.org/anabases/4379

DOI : 10.4000/anabases.4379

ISSN : 2256-9421

Éditeur

E.R.A.S.M.E.

\section{Édition imprimée}

Date de publication : 1 octobre 2013

Pagination : 246-257

ISSN : 1774-4296

\section{Référence électronique}

Magali Soulatges, «Les thrillers de Daniel Chavarría, ou la rencontre de l'érudition classique et du polar cubain », Anabases [En ligne], 18 | 2013, mis en ligne le 01 novembre 2016, consulté le 20 octobre 2019. URL : http://journals.openedition.org/anabases/4379 ; DOI : 10.4000/anabases.4379

Ce document a été généré automatiquement le 20 octobre 2019.

(c) Anabases 


\title{
Les thrillers de Daniel Chavarría, ou la rencontre de l'érudition classique et du polar cubain ${ }^{27}$
}

\author{
Magali Soulatges
}

\section{L'F્Fil de Cybèle : le vocable gréco-latin entre étymologie savante et (ré)invention ironique ${ }^{28}$}

L'histoire se déroule à Athènes, entre l'« été de l'archonte Cratès " et l'« été d'Épaméinon » (434/33-429/28 av. J.-C.), dernières années du pouvoir de Périclès et prémices de la guerre du Péloponnèse, dans une ville grouillant de marginaux en tous genres: danseuses callipyges déclassées, magiciennes louches, prostituées ambitieuses, rufians sans scrupules, affranchis cupides, esclaves déterminés... Et un mendiant métèque. Un illuminé persuadé d'avoir été élu par Cybèle pour venir fonder à Athènes le plus grand sanctuaire jamais consacré à la déesse ainsi qu'une nouvelle religion régie par un culte à mystères, l'Église de la Somme. Associé à une courtisane déchue et à une hétaïre épileptique encline aux visions mystiques, toutes deux dévouées à leur divinité tutélaire Aphrodite Pandémos, l'émissaire autodéclaré de cybèle se heurte très vite aux grands de la cité (stratèges, magistrats, clergé), qu'inquiètent son credo dérangeant et son prosélytisme efficace. Ce d'autant que le trio interlope serait impliqué dans un autre mystère : la disparition d'une pierre sacrée, «l'œil de Cybèle », dérobée au Parthénon sur une statue de la déesse et convoitée pour des raisons diverses par à peu près tout le monde. Plusieurs "déchiffreurs d'énigmes" se lancent en même temps dans l'enquête, au cœur d'une Athènes de bruit et de fureur où l'ambition sans frein et la course au pouvoir semblent seules gouverner les individus et leurs actes.

1 Interrogé par Édouard Waintrop à l'occasion de la parution française de L'Fil de Cybèle $(1997)^{29}$, Daniel Chavarría relie le choix du polar historique, nouveau pour lui, à plusieurs éléments. Pour l'helléniste formé sur le Vieux Continent aux humanités classiques, puis Cubain d'adoption, l'analogie s'impose entre la santeria pratiquée dans l'île et la religion grecque classique, en raison d'une même finalité pragmatique et économique; quant à la part mystique du sentiment religieux, très présente dans le 
culte Yoruba dont procède la santeria, on la retrouve dans les rites d'initiation au cœur des cultes orientaux à mystères concurrents de la religion grecque officielle, tels les mystères d'Éleusis, les religions orphiques, ou encore le culte phrygien de Cybèle-Rhéa. L'Athènes du romancier ne ressemble guère à l'image de la Grèce $d u$ ve siècle véhiculée par l'école: «J'ai aussi voulu désacraliser la culture grecque, explique Chavarría, lui redonner sa chair, rendre la beauté simple de cette civilisation et de sa philosophie». $\mathrm{Ce}$, en "retrouv[ant] la puissance réelle de la langue d'Aristophane", trop souvent censurée par les traducteurs modernes imperméables à sa "saine obscénité ", et en mettant en lumière les attitudes parfois très "modernes » de personnalités hors du commun comme Périclès, Aspasie ou Alcibiade. Enfin, l'écrivain rompu au néo-polar se dit fasciné par cet underground social que forment esclaves, mendiants et prostituées en marge de la citoyenneté grecque, monde exposé et précaire dont le quotidien se résume souvent à une lutte sans merci pour la survie. Et Chavarría de lever toute ambiguïté sur le sens de sa démarche: «Je ne théorise pas, je montre la Grèce comme je la comprends. "

2 Par les milieux décrits et les personnages qui les arpentent, en même temps que par l'alliance originale du roman policier, de mœurs et érotique, L'Æil de Cybèle répond en fait au souhait de l'auteur de dessiner une nouvelle catégorie narrative du polar, produit syncrétique d'influences littéraires diverses : la picaresca cubana ${ }^{30}$. Au point que le plus éloigné, par son arrière-plan historique, de La Havane d'aujourd'hui apparaitrait presque comme le plus "cubain» des romans de Chavarría, en prélude à Adiós muchachos dont l'héroïne moderne, qui tient commerce actif de ses avantages, semble hériter en droite ligne de Lysis de Milet, «transformant ses éphémères tremblements de callipyge en argent pour le marbre perdurable de la Somme ${ }^{31}$ ». L'Eil de Cybèle n'en relève pas moins d'abord du polar historique, tel que ce sous-genre du roman policier se trouve illustré de manière emblématique, à partir des années 1980, par Le Nom de la rose, tel aussi que prisé par les auteurs latino-américains ${ }^{32}$. Si son principe en est simple (une intrigue policière située dans le passé), son dispositif narratif fait montre, lui, d'une complexité toute "chavarrienne", tant sur le plan proprement narratologique que sur celui de l'énonciation: au récit-collage, qui juxtapose voire imbrique des formes narratives hétérogènes (épistolaire, fable, dialogue philosophique, chronique historique, anecdote...), répond ainsi une mosaïque de régimes discursifs eux-mêmes variés (récit, dialogue, monologue intérieur, polyphonie, digressions...), des jeux d'analepse et de prolepse narratives venant se surajouter encore à un ensemble déjà bien embrouillé.

3 Et si là résidait, dans cet habile montage confinant au puzzle inextricable, l'un des enjeux génériques les plus originaux et les plus stimulants de L'Eil de Cybèle? Roman historique, L'Eil de Cybèle inscrit son intrigue dans un contexte précis, Athènes au temps de Périclès, cédant à une loi du genre : la reconstitution « documentaire » d'une époque et de ses mœurs, la précision du tableau conditionnant l'acceptation par le lecteur du décentrage spatio-temporel postulé par le pacte de lecture. Or en même temps qu'elle est située et scrupuleusement documentée, l'« histoire grecque » racontée par Chavarría, qui relève conjointement du polar, se présente sous une forme peu lisible, par fragments, comme démontée en autant de pièces énigmatiques d'un puzzle narratif à reconstituer, alors que la pensée spéculative est déjà sollicitée pour enquêter sur le contenu-même de "l'affaire Cybèle»: sur quoi exactement, de l'améthyste sacrée disparue ou d'Athènes comme énigme historique, le lecteur est-il 
donc invité à investiguer? Loin de fournir seulement l'ancrage d'un polar historique, la Grèce classique de Périclès, telle qu'exposée en pièces détachées dans L'Eil de cybèle, semble ainsi pour le lecteur une autre (la véritable ?) énigme à résoudre; et les mots, grecs essentiellement, la clef indispensable pour entrer dans le secret d'Athènes, à l'instar du métragyrte de Cybèle obnubilé par la langue à connaître pour pénétrer le mystère de la Somme.

\section{Construire un monde, le plus meublé possible 33}

Dans son Apostille au "Nom de la rose ", Umberto Eco définit les trois façons pour un auteur de roman historique d'inscrire une fiction dans le passés ${ }^{34}$. Il peut s'agir de brosser à grands traits une toile de fond donnant "l'impression d'éloignement historique $^{35}$ » et offrant un prétexte simple à l'écriture. Un passé historique plus travaillé et qui tend vers la reconstitution " archéologique » renforcera, lui, l'effet de réel, sans brider pour autant les droits de l'imagination même si, le concède Eco, les éléments historiques posés réduisent l'éventail des possibles narratifs à proportion de la recevabilité de ceux-ci, de leur vraisemblance: traditionnellement, cet équilibre passe par l'inscription dans le roman de personnages historiques auxquels sont prêtées actions fictives et pensées extrapolées. Troisième cas de figure : la reconstruction du passé à partir de personnages inventés conçus comme une incarnation symbolique des mouvements profonds d'une époque ou de l'histoire, et «dessin[ant] le processus par lequel [les] causes ont évolué lentement pour produire leurs effets ${ }^{36} »$. L'Eil de Cybèle joue sciemment de ces trois tendances de la fiction historique, combinées comme pour mieux emporter (égarer?) le lecteur dans une narration qui oscille entre la pochade facétieuse " à l'antique ", à effets faciles, et la leçon métaphysique portée par des types symbolisant un groupe ou une condition, en passant par la démonstration historiographique qu'informent de solides connaissances, la frontière entre fiction et histoire n'étant pas toujours ici marquée.

C'est une constante chez les auteurs de fictions historiques, romans ou polars, que de souligner l'importante tâche de recherche documentaire menée en amont de l'écriture, tâche néanmoins facilitée quand une familiarité de longue date existe avec la période décrite. Les liens de Chavarría avec la Grèce antique relèvent du compagnonnage intellectuel ininterrompu depuis l'enfance montévidéenne bercée par les mythes de l' Iliade et de l'Odyssée jusqu'au professorat de grec à l'université de La Havane : soient quelque cinquante ans d'immersion dans un monde approché d'abord par le biais du déchiffrement ludique puis exploré en chercheur autodidacte insatiable, avant de devenir l'objet d'une réinterprétation personnelle à la faveur de l'expérience révolutionnaire cubaine. C'est dire si la Grèce classique du romancier témoigne d'une proximité quasi empathique; et c'est dire l'évidence avec laquelle s'imposent les realia qui «meublent » L'Eil de Cybèle, «modelant» un lecteur assez vite "complice» de l'étendue du savoir chavarrien, quand un certain découragement eût pu naître d'une trop grande distance intellectuelle avec l'auteur ${ }^{37}$. De ce point de vue d'ailleurs, le roman mérite l'estampille de «thriller d'érudition », avec ce pari paradoxal de viser un large public tout en soumettant le lecteur à « une certaine discipline pénitentielle ${ }^{38}$ ».

6 À ce lecteur acquis ou gagné, L'Cil de Cybèle offre de fait plus qu'il n'en attendrait, par l'omniprésence et l'ampleur de la référence encyclopédique : l'on devine par exemple que la narration de la captivité puis de la mort de Xanthe le Lycien dans les mines du 
Laurion doit autant à Thucydide, Xénophon, Plutarque ou encore Aristote ${ }^{39}$ qu'à une connaissance concrète des lieux par l'archéologie. Copieuse et ouverte, sans souci de hiérarchie des sources utilisées, la référence antique intervient à des degrés divers, de la mention ponctuelle à l'exposé structuré, sans jamais tomber dans le piège de l'essai déguisé. Digérée par l'historien, elle réapparaît sous la plume du romancier selon deux modalités privilégiées : la dissémination et la réécriture, jusqu'au pastiche parfois. Si un tableau assez complet du statut sociologique de la femme à Athènes au v viècle se dégage ainsi, c'est à partir de la juxtaposition de croquis rapides d'esclaves discrètes, de descriptions plus étoffées de la vie d'une concubine, de portraits en creux d'une hétaïre convoitée par un notable de la cité; et les divers banquets qui jalonnent le roman peuvent être regardés comme autant de relectures de Platon mises au service d'une reformulation personnelle, démarquée du philosophe, du paradigme du banquet classique. L'érudition à l'œuvre paraît inépuisable, déboutant par avance le lecteur tatillon, public fréquent des romans historiques, qu'un inventaire de ces realia tenterait pour en pointer les omissions ou les inexactitudes; pour autant, elle ne recherche pas l'exhaustivité, et les silhouettes dessinées ne portent jamais plus qu'un simple chiton ou himation - au demeurant, l'unique voile transparent en lin safran de Lysis ne suffit-il pas à «habiller» l'hétaïre sensuelle ?... «Que le lecteur pointilleux se reporte à ses encyclopédies", répond Chavarría à ce public toujours envisageable: "Le lecteur indulgent acceptera que [...] tous mes personnages historiques sont fidèles à Hérodote, Thucydide, Platon, Xénophon et à l'historiographie postclassique ${ }^{40}$. "

7 Le monde de L'Æil de cybèle, "meublé " avec une minutie érudite mais de façon sélective, n'en est donc pas moins un monde incomplet, avec ses manques accueillants à l'investigation policière. Car la Grèce historique se modèle avant tout sur la démarche romanesque, orientée vers une idée-clef, l'interrogation du lien organique unissant religion, sexe, argent et politique dans une société en crise traversée de contradictions ; mais plus encore peut-être, sur le plan métalittéraire, orientée vers la question de la transtextualité comme ressort esthétique du roman noir, en particulier du polar historique. L'on notera que cette transtextualité peut intégrer l'histoire contemporaine comme un texte propre, superposable aux autres, en une formule romanesque audacieuse alliant polar historique et néo-polar, pourtant théoriquement incompatibles. Reprenons l'exemple de Xanthe le Lycien, torturé à mort au fond d'une caverne du Laurion par les hommes de main de Nicias : aux références déjà signalées se mêle à l'évidence un texte moderne, impossible à écrire à Cuba dans les années 1990 selon Justo Vasco ${ }^{41}$, portant sur l'histoire "psychologique » d'une dictature et de ses tortionnaires, dictature à maints égards analogue à l'exploitation par Nicias, dans des conditions totalement inhumaines, de sa concession minière. Moyennant quoi, inscrite dans un « feuilletage » très élaboré du texte, la référence antique convoquée dans L'Æiil de Cybèle a pu paraître à l'éditeur du roman assez peu intelligible pour que Chavarría en explicite le fonctionnement au lecteur. Comme souvent avec les policiers historiques, un paratexte explicatif accompagne donc le roman, bien que l'auteur ne croie guère pour sa part à l'utilité d'un tel dispositif ${ }^{42}$ :

Lorsque j'ai donné le manuscrit à lire, on m'a demandé d'y ajouter des notes pour orienter le lecteur. Quelqu'un m'a objecté que le mélange de la fiction avec des faits et des personnages historiques rendait difficile la distinction entre réel et imaginaire. Et certains n'ont pas manqué de suggérer que je manipulais les personnages historiques pour les ajuster à mes buts littéraires.

Je ne suis pas d'accord pour transformer ce roman en texte rempli de notes didactiques. Mais comme ces avis m'ont inquiété, j'ai décidé d'adjoindre deux 
annexes. On trouvera à la fin des cartes et un lexique. Certaines des choses qui n'y figurent pas sont le résultat de ma propre invention. Ainsi le lecteur pourra se faire une idé $\mathrm{e}^{43}$.

El Ojo Dindymenio paraît la même année (1993) à Mexico, Montevideo et Barcelone ${ }^{44}$. L'édition uruguayenne est complétée par un épais Guía de lectura y léxico de la novela de 106 pages, non traduit lors de la parution en France du roman dans la collection de poche Rivages/noir (1997); guide composé d'une courte présentation de la Grèce classique avec références bibliographiques et d'un lexique détaillé. Le mode d'emploi du roman varie ainsi selon l'aire culturelle à laquelle il s'adresse, pour des raisons semblables à celles invoquées par U. Eco lors de la réédition en 2011 du Nom de la rose : une connaissance différente par le public, selon les pays, de la culture antique ${ }^{45}$. Cependant, si les deux versions du roman (en espagnol et en français) diffèrent par la présentation et le volume du paratexte, ce dernier reste composé du même type de pièces : deux «notes de l'auteur ", en ouverture et conclusion du roman dans l'édition française, et les annexes déjà mentionnées, soient deux fonds de cartes (Grèce, Golfe Saronique) utiles à la compréhension des nombreux toponymes et gentilés présents dans le texte, et le lexique ${ }^{46}$. Note liminaire et note en postface réunissent des informations traditionnellement données par les auteurs en marge des romans historiques, sur la genèse de l'œuvre (ici expliquée par deux références ponctuelles extraites d'un manuel anglais d'études grecques et d'une monographie française sur les communautés religieuses antiques, ouvrages tous deux $\mathrm{du}_{\mathrm{XIX}} \mathrm{e}^{\mathrm{e}}$ iècle ${ }^{47}$ ), sur l'invention de tel personnage (celui du métragyrte de Cybèle ici), et sur la présence-même d'un paratexte explicatif : « Il s'agit d'une courtoisie à l'adresse des lecteurs si particuliers de romans historiques, qui ont l'habitude d'utiliser la littérature comme source d'information", soutient Chavarría. Ce qui conduit le romancier à s'expliquer, brièvement, sur un point tout aussi attendu: sa fidélité plus ou moins grande aux leçons de l'histoire, qui se résume ici à trois légères entorses à la chronologie de Thucydide structurant la temporalité de l'intrigue, et un anachronisme franc avec l'insertion au dernier chapitre du roman d'un palindrome chrétien postérieur au v siècle. Et Chavarría de conclure « qu'un bon roman trouve [toujours] en lui-même son explication. Et s'il n'est pas bon, à quoi sert-il d'y adjoindre des explications?»

\section{Pour le dire grecquement ${ }^{48}$}

9 Parce qu'elle met en regard deux époques au moins, toute fiction historique se construit par nature sur un porte-à-faux, renforcé dans le cas du polar par la projection sur un temps antérieur d'une perception moderne, informée par leur histoire respective, de la justice, de la police et des méthodes d'enquête criminelle ${ }^{49}$. S'y ajoute l'orientation particulière que le polar historique adopte lorsqu'il intègre, comme L'Cil de Cybèle, plusieurs traits du néo-polar : réalisme sans concession, peinture de milieux sociaux populaires, éclairage mis sur les violences urbaines, dénonciation des dérives de la société... Deux écueils pouvaient faire manquer son but à Chavarría, novice dans le genre du polar historique. Du fait d'une référence antique très documentée et prégnante, la donnée policière aurait pu tomber dans le défaut de l'intrigue plaquée, vice dont souffrent bien des polars historiques dans lesquels la formule, en particulier "à l'antique ", ne parvient pas à "prendre ". Ou bien, l'érudition prenant le pas sur le thriller au détriment du rythme (celui de l'action comme celui de la lecture, que peut embarrasser un imposant appareil de notes), le roman aurait pu tourner à l'abstraction 
«défictionnalisante » pour happy few, à la limite de la lisibilité - un objet comme La Caverne des idées de José Carlos Somoza doit, nous semble-t-il, une part de son caractère ardu et déroutant à l'aspect quasi expérimental de son écriture. Mais Chavarría transforme l'essai, en faisant preuve d'une extrême habileté dans le maniement de l'intertextualité savante et l'intégration de la langue grecque à l'intrigue, caractéristiques du roman. Par ce double biais, le monde «meublé » de L'Æil de cybèle acquiert une indiscutable épaisseur, jusqu'à fonctionner de façon autonome grâce à un efficace système de renvois internes (par comparaisons et jeu d'anaphores/cataphores) donnant l'illusion d'une existence bien réelle de la cité athénienne. L'on comprend ici tout le bénéfice pour l'auteur à pouvoir s'adresser à un public européen nourri par la culture classique, pour qui un appareil de notes didactiques ne s'impose pas, ou dans une moindre mesure, à la différence du lectorat sud- ou nord-américain qu'il faut accompagner en " pédagogue " avec un Guía de lectura y léxico de la novela... Et sans doute est-ce la raison pour laquelle le paratexte de l'édition française se révèle assez peu opérant, avec son refus manifeste de citer des sources ou de "traduire» la civilisation grecque, et avec son lexique réduit a minima ${ }^{50}$.

Il reste que dans le même temps, L'Æil de Cybèle se présente comme une brillante démonstration sur l'idée-même de "référence antique", dans laquelle Chavarría développe une poétique réfléchie de la citation (entendue dans le sens dynamique d'importation d'un « texte autre » dans un discours premier). La question se rattache à la problématique plus large de la langue d'écriture du roman historique ${ }^{51}$, plus délicate encore lorsqu'il s'agit d'ancrer la fiction dans les temps reculés de l'Antiquité : indépendamment en effet $\mathrm{du}$ fondement éthique voire idéologique de tout positionnement personnel vis-à-vis du passé (que l'on peut vouloir « restaurer » jusque dans ses mots, ou au contraire tenir pour définitivement inactuel), la question est bien celle d'une restitution au plus près, ici par la langue, de l'univers narré. Et donc, d'une décision esthétique. Retrouver un « langage d'époque » étant d'un côté inenvisageable, ne serait-ce qu'en raison des significations perdues de nombre de mots ${ }^{52}$, et de l'autre une langue contemporaine pouvant gêner par son caractère anachronique, c'est le plus souvent derrière une solution de compromis que les auteurs se retranchent, optant pour une langue moderne neutre, émaillée de mots et de tournures "d'époque » ou créant l'illusion de cet éloignement temporel. Au demeurant, ce qui pourrait troubler l'historien soucieux de reconstitution archéologique embarrasse rarement le lecteur de fiction, habitué aux conventions liminaires des pactes de lecture romanesque.

11 La solution adoptée par Chavarría surprend au premier abord, avant de séduire par son ingéniosité. Le dispositif romanesque de L'Eil de Cybèle, nous l'avons dit, fait voler en éclats l'homogénéité de principe d'une narration historique par la fragmentation des deux instances respectives du récit et du discours en un texte-puzzle et un collage énonciatif. Or il se trouve que le discours tend souvent à recouvrir le récit, au point que le roman pourrait tout à fait être lu/écouté comme une composition polyphonique, chaque fragment narratif (qui épouse grosso modo le découpage des 65 chapitres) donnant à entendre une " voix ", directe ou empruntée : si le lecteur est ainsi confronté sans médiation à quelques personnages-clés du roman grâce à la présence de nombreux monologues intérieurs et discours indirects libres, d'autres voix lui sont également audibles, médiatisées elles, à travers par exemple les pastiches (imitation de Plutarque pour narrer la "vie de Nicias", imitation des lógoi hérodotéens pour l'histoire du Scythe...). Quoiqu'à des degrés divers - les pensées secrètes du métragyrte renseignent 
plus sur le personnage que l'imitation de son écriture ne permet d'approcher l'intimité d'Hérodote -, chacune de ces voix se trouve donc corrélée à une subjectivité (et à une focalisation, au plan narratologique), et intégrée à un dispositif énonciatif général caractérisé par son hétéroglossie. Comment, par ce droit reconnu de tous à la parole, mieux assurer la promotion démocratique, dans l'espace de la pólis, de ces marginaux qui en sont aussi la voix, fût-elle romanesque? À chaque discours sa langue, par ailleurs : en imaginant une sorte d'idiolecte "naturel » pour chaque personnage/voix et en laissant travailler par eux-mêmes au fil de l'intrigue policière ces langages qui se cherchent, se fourvoient, s'amendent, se censurent, se dissimulent, se livrent..., Chavarría semble vouloir faire de l'expression d'une coïncidence à soi dans et par la langue un préalable au discours de vérité qui sous-tend toute enquête criminelle. Partant, l'« archilangue » (pour parler comme le métragyrte de Cybèle...) dans laquelle est rédigé le roman se trouve dispensée d'inférer sa plausibilité d'une cohérence d'ensemble puisqu'elle se donne comme un texte par nature hétérogène. Hétérogène, et hybride: car le grec ancien qui s'y glisse fait nécessairement figure de «langue étrangère » dans le récit alloglosse en espagnol ou en français.

«Parler grecquement » repose dans L'Eil de Cybèle sur une variété impressionnante de stratégies d'intégration de la référence antique (mot ou idée) dans un récit-cadre qui confine par moments au texte métissé. La citation concerne aussi bien des mots simples que des unités plus complexes (expressions, fragments textuels, réécriture d'ensembles plus vastes), insérés dans le texte sous une forme explicite (citation au sens strict) ou dérivée (tournures stylistiques, registres, allusion intertextuelle...). Cette présence est marquée ou non typographiquement (italiques pour les emplois en mention ou certains concepts abstraits, graphies translittérées avec leur orthographe et leur accentuation particulières). Assez souvent, le roman érudit dissimule l'origine de ses éléments importés derrière une manière "à l'antique " susceptible de contenter aussi bien le lecteur cultivé (capable de remonter par lui-même jusqu'à la référence) que le lecteur astucieux ayant compris les règles du jeu. Sont touchés par les stratégies proprement linguistiques d'insertion du mot grec les trois niveaux grammaticaux de la morphologie (simplification de certaines graphies, adaptation des flexions), de la syntaxe (style) et du lexique - celui qui figure en annexe de l'édition française, pourtant réduit par rapport à son équivalent espagnol, comprend quelque 300 entrées, noms communs et noms propres confondus. Le plus souvent, ces insertions opèrent par simple dilution dans la syntaxe d'accueil. Enfin et surtout, idées et mots grecs importés peuvent faire l'objet d'une glose, immédiate ou différée dans le texte (définitions sommaires, paires lexicales, équivalents latins, traductions ou pseudo-traductions, comparaisons...). La mise en œuvre d'un tel éventail de procédés pourrait faire craindre la confusion. Pourtant, l'interférence des deux systèmes de discours (grec/espagnol ou français) ne rend jamais illisible cette hétérogénéité linguistique constitutive du texte métissé. De même, n'est pas remise en question son acceptabilité par le lecteur, lequel répond au contraire ici, par sa compétence «bilingue ", à l'image du « lecteur modelé » souhaité par Eco.

13 L'intégration de la référence antique dans le récit obéit à un premier enjeu romanesque, attendu: «redonner [leur] chair ${ }^{53}$ " aux realia de la Grèce du ve siècle convoquées par le roman historique. Pour assurer cette "illusion romanesque ", L'Eil de Cybèle emprunte deux directions en apparence opposées. D'une part, elle recherche l'effet de réel en allant au plus près du monde peint, désigné à partir de ses mots originaux, application directe de la démarche de Périclès et Alcibiade obstinés à 
" appeler les choses par leur nom ${ }^{54}$ ». Mais en même temps, instaurant une distance avec ces choses correctement quoique «bizarrement » nommées, elle introduit dans le tableau un effet d'étrangeté, comme au moment de l'enclenchement du procès de Nicias, où l'accumulation des termes grecs déréalise presque la situation - le but étant aussi, par ce moyen, de ménager un climat de suspense. Ce double mouvement, en fait complémentaire, de banalisation et de soulignement déréalisant de la référence antique, est particulièrement sensible dans les passages qui entendent restituer une oralité spontanée. Chavarría pioche là dans une batterie fournie de procédés créateurs d'une fiction de langue orale, avec une prédilection affichée pour le langage brut et cru de l'injure ou inspiré des comédies d'Aristophane, dont le romancier se proposait ailleurs de régénérer la traduction. De façon plus originale, Chavarría s'attache à « ressusciter » le caractère phonique et prosodique de la langue grecque, en déléguant pour partie cette tâche incertaine au métragyrte de Cybèle, en quête constante du ton et de la cadence justes pour psalmodier l'indicible de la Sainte Triade ${ }^{55}$. Mais c'est par le discours métalinguistique tenu sur la langue ancienne que L'Eil de Cybèle se distingue peut-être le plus des autres romans de sa catégorie : par la promotion narrative du métissage linguistique (dans la forme bilingue privilégiée par le récit, et en tenant compte de la diversité linguistique de l'oekoumène cartographiée en annexe du roman), tout le récit en effet parait vouloir ériger la polyglossie en valeur, culturelle de vecteur d'une communication universelle, et esthétique parce que matériau premier de la poïsis.

Second enjeu romanesque des stratégies d'insertion de la référence antique : alimenter le jeu verbal placé au cœur de l'ironie drolatique développée par le roman conjointement au propos sérieux. Ce jeu verbal vaut autant pour lui-même que parce qu'il est mis au service de la « désacralisation » de la culture grecque classique visée par Chavarría : dans les deux cas, l'ironie propose donc un ancrage du texte dans le présent, celui de l'auteur amateur de facéties littéraires, celui de l'érudit conduit par les propres realia de son époque à réévaluer ses savoirs acquis et les leçons orientées de ses humanités d'hier. Aussi cette ironie s'exprime-t-elle en priorité à travers la catégorie de la discordance, usant et abusant des effets de ruptures et autres décalages narratifs et linguistiques censés saisir in vivo la " discordance des temps »; au point que tous les procédés utilisés (d'esprit parfois très oulipien) peuvent être considérés comme jouant toujours sur un anachronisme. L'inventivité de Chavarría dans ce domaine parait sans limites, qui témoigne d'un goût prononcé pour les jeux lexicaux: jeux avec des archaïsmes (abigéat), des néologismes - mais le grec ancien n'est-il pas déjà friand de néologismes ? -, des étymologies revisitées à partir de leurs potentialités narratives un archer scythe précis dans ses tirs est aussitôt rebaptisé « divin hypermétrope »-, des dérivations parodiques (archi-mensonge), des épiclèses créées de toutes pièces (la Milésienne aux Fesses Trépidantes)... Ce dernier exemple montre que le jeu est aussi plus largement pensé comme un exercice de style - tout l'arsenal des figures issu de la rhétorique antique est alors convoqué -, avec le pastiche d'un genre ou d'une œuvre comme horizon, sur le mode ludique mais aussi sérieux. L'intertextualité joue là à plein, plus ou moins signalée, l'effet caustique découlant des modes d'insertion de la référence dans le récit-cadre : directe et ponctuelle (comme avec ce vers tragique de l' Hippolyte d'Euripide mis dans la bouche sottement mélodramatique de Lysis : «ô jour odieux, odieuse lumière que je ne verrai plus »), indirecte et disséminée dans le texte (comme avec l'écriture épique homérique, imitée sans que l'on sache toujours si l'on a affaire à une traduction personnelle, une pseudo-traduction, ou une invention). 
Combinés et empilés, tous ces procédés engendrent parfois des moments de pure hilarité, que n'auraient certes pas boudés les spectateurs d'Aristophane : telle apparaitt l'apokálypsis burlesque du Sommet de la Somme, "anarchiste couronné ${ }^{56}$ " sous les auspices conjoints du Mónos et de la déesse Équivoque, dont Chavarría ne parle évidemment pas, mais qui scénographie de bout en bout cette transfiguration sacrée du myste par la religion et le sexe. L'on est fondé, pour le coup, à douter de la virginité littéraire postulée chez le lecteur de L'Eil de Cybèle, pour qui le roman «trouve[rait] en lui-même son explication » : car n'est certainement pas le premier candide venu celui qui peut lire dans tout son foisonnement ce texte profondément hybride et tourné vers une mise en scène poétique de cette hybridité. De ce lecteur en effet, même « modelé " par le roman, n'est-il pas attendu une somme intimidante de compétences, policière d'abord, mais aussi linguistique, historique, politique, religieuse, culturelle, littéraire, ludique, poétique...?

"Je crois que les gens aiment les polars non parce qu'il y a des assassinats ni parce que l'on y célèbre le triomphe de l'ordre moral (intellectuel, social, légal et moral) sur le désordre de la faute. Si le roman policier plaît, c'est qu'il représente une histoire de conjecture à l'état pur ${ }^{57}$ ", avance Eco pour définir ce qu'il appelle la «métaphysique policière ». En plaçant au centre du récit un personnage appelé Hypothèse ${ }^{58}$, L'Æil de Cybèle ne contredit guère cet axiome, que vient corroborer une intrigue peu soucieuse de faire reposer l'investigation sur l'examen d'indices matériels et de preuves. Caractéristique du polar moderne, voire du néo-polar, la question du mobile de l'acte criminel ("pourquoi a-t-on tué/volé ?») prend le pas sur celle de son auteur («qui a tué/volé ? »); et c'est à un récit éclaté, polyphonique et hybride qu'est ici déléguée la fonction d'enregistrer la progression de l'enquête, non à un détective particulier chargé d'en centraliser et synthétiser les résultats. D'où peut-être ce tableau romanesque hétéroclite, presque didactique, des différentes méthodes et techniques d'investigation criminelle possibles pour élucider un mystère en l'absence d'enquêteur désigné : méthode intuitive, fondée sur l'observation et le bon sens, appliquée avec pragmatisme par le perspicace Agathyrsos; méthode judiciaire, bénéficiant du soutien de l'appareil d'État et garantissant la procédure légale d'un procès - c'est la méthode de Périclès accusant publiquement Nicias devant les généraux du Stratègéion; méthode musclée, pratiquant l'interrogatoire avec les outils de la perquisition et de la torture la fouille sauvage de la maison d'Alcibiade par Lysis se rapproche de la perquisition illégale, et l'on ne reviendra pas sur les conditions de la mort de Xanthe le Lycien dans les mines du Laurion; méthode philosophique, variante rationnelle et non-violente de l'interrogatoire, " posant des questions et y répondant comme dans la maïeutique de Socrate ${ }^{59}$ »; méthode sémiotique, de déchiffrement de signes plus ou moins directement liés au crime, envisagée dans une version surtout religieuse - c'est Nicias le superstitieux, obsédé par l'interprétation des augures, et appointant des devins privés convaincus pour leur part que «dans l'exercice de la divination, la première chose est de deviner ce que désire le consultant ${ }^{60} »$.

Quelle que soit leur efficacité respective supposée, de fait plutôt mise en doute par le récit, ces diverses approches de l'enquête trahissent toutes la même obsession : mettre un nom, le plus approprié possible, sur les choses (sur le voleur, sur le lieu de recel de «l'œil de Cybèle »...) et partant, être assuré que ces choses font l'objet de la bonne lecture. Interroger, puis nommer, est ainsi étroitement lié au maniement des signes linguistiques et à une démarche d'appropriation de leur sens, ce qu'illustre avec ironie la recherche par le métragyrte de Cybèle de son ónoma liturgique officiel : «Oui, Atys de 
Pamphylie deviendrait le premier prêtre de la nouvelle Église. Mais comment disait-on Premier Prêtre en grec? Prostate? Protoprêtre ? Hieratkós Archon? [...] Il essaya des titres en phrygien, en lydien, en carien, mais il se rendit compte que si la nouvelle Église était fondée à Athènes, il lui fallait se trouver un nom grec ${ }^{61}$.» Or le réel, loin de se plier aisément à l'acte simple de nomination, se révèle au contraire fondamentalement polysémique, et ses signes ne laissent deviner aucun ordonnancement naturel du monde ; au mieux doivent-ils être considérés comme des indices, traces imparfaites et lacunaires d'une autre réalité appelant toujours le déchiffrement.

17 C'est de manière quasi maladive et tête baissée que les personnages de L'Fil de Cybèle se jettent dans cette enquête-là, dont l'affaire de l'améthyste dérobée apparaît vite un épiphénomène, ou un symptôme. Symptôme de la situation d'Athènes à la fin du siècle de Périclès, instable au plan religieux, incertaine politiquement, menacée économiquement, vulnérable socialement. Mais qui a volé "l'œil de Cybèle», et pourquoi ? La cité entière, répond le roman, qui révèle qu'à peu près tout le monde a trempé à un moment donné dans le vol de la pierre sacrée; et ce, afin que soient réaffirmés les lois de la pólis et le «nom » de citoyen. Apparemment tout au moins, car Cybèle la Phrygienne, déesse énucléée telle đEdipe, rapatrie toujours l'affaire policière dans la sphère théologique d'une histoire sacrée, d'une parabole hissant l'enquête sur le nom au rang d'un destin. Un mythe, en quelque sorte.

\section{NOTES}

27. Le présent atelier de Magali Soulatges est bien le $6^{\mathrm{e}}$. C'est pas erreur que l'atelier paru dans le $\mathrm{n}^{\circ} 17 \mathrm{~d}^{\prime}$ Anabases porte le $\mathrm{n}^{\circ} 4$ au lieu du $\mathrm{n}^{\circ} 5$.

28. Le premier volet de cette étude est paru dans Anabases 16 (2012), p. 285-293 : "L'écriture policière : une forme ouverte aux affleurements de la référence antique ». Nous recourons dans cette étude à l'édition française de L'Æil de Cybèle, Paris, Payot \& Rivages/noir, 2011.

29. "Chavarría, label hellène », Libération, 18 décembre 1997, en ligne : http://www.liberation.fr/ livres/0101231761-chavarrria-label-hellene-l-uruguayen-de-la-havane-a-situe-l-action-de-sondernier-polar-dans-l-athenes-du-siecle-de-pericles-entretien-avec-un-helleniste-nonconformiste-daniel-chavarria-l-oeil-de-cyb (consulté le 10 mai 2013).

30. M. SOULATGES, « L'ÉCRITURE POLICIÈRE... », P. 292.

31. D. CHAVARRÍA, L'EIL..., P. 393.

32. U. ECO, IL NOME DELLA ROSA, MILANO, FABBRI-BOMPIANI, 1980 (TRAD. FRANÇAISE PAR J.-N. SCHIFANO : PARIS, GRASSET ET FASQUELLE, 1982). UNE MISE AU POINT SUR LE GENRE DU ROMAN POLICIER HISTORIQUE, QUI RESTE RELATIVEMENT BOUDÉ DES ÉDITEURS (MALGRÉ LA COLLECTION PHARE « GRANDS DÉTECTIVES » DE J.-CL. ZYLBERSTEIN, CHEZ 10/18), EST PROPOSÉE PAR L. BROCHE ET J.-CHR. SARROT DANS UN RÉCENT ESSAI, LE ROMAN POLICIER HISTORIQUE. HISTOIRE ET POLAR: AUTOUR D'UNE RENCONTRE, PARIS, NOUVEAU MONDE ÉDITIONS, 2009 (BLOG ASSOCIÉ: HTTP :// WWW.POLAR-HISTORIQUE.COM/).

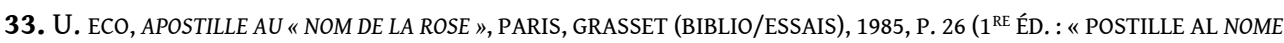
DELLA ROSA », ALFABETA 49, 1983).

34. U. ECO, APOSTILLE..., P. 85-87. 
35. "J'ai pratiqué à la manière d'un dentiste ", entretien de M. Bono avec U. Eco, Libération, 17 septembre 2011, en ligne: http://www.liberation.fr/culture/01012360342-j-ai-pratique-a-lamaniere-d-un-dentiste (consulté le 10 mai 2013).

36. U. ECO, APOSTILLE..., P. 89.

37. Le concept de "lecteur modelé », opposé à celui de « lecteur empirique », est théorisé par U.

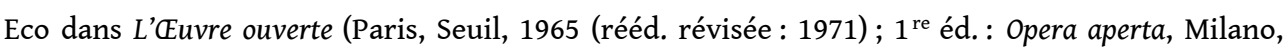
Bompiani, 1962) puis Lector in fabula (Paris, Grasset, $1979 ; 1^{\text {re }}$ éd. : Milano, Bompiani, 1979). Dans Apostille au "Nom de la rose», U. Eco revient sur le sujet pour répondre à la question du lecteur postulé par ce roman exigeant : «Quel lecteur modèle voulais-je quand j'écrivais ? Un complice, bien sûr, qui joue mon jeu. Je voulais devenir complètement médiéval et vivre le Moyen Âge comme si c'était mon époque (et vice versa). Mais en même temps, je voulais de toutes mes forces que se dessine une figure de lecteur qui, après avoir surmonté l'initiation, devienne ma proie ou la proie du texte et pense ne plus vouloir autre chose que ce que le texte lui offrait. »

38. U. ECO, «J'AI PRATIQUÉ... ».

39. Respectivement dans La Guerre du Péloponnèse, les Poroi, la Vie de Thémistocle et la Vie de Nicias, et la Constitution d'Athènes.

40. D. CHAVARRÍA, L'EIL..., « NOTE DE L'AUTEUR », P. 10.

41. M. SOULATGES, « L'ÉCRITURE POLICIÈRE... », P. 290.

42. La même conviction anime U. Eco, dont la réédition en 2011 du Nom de la rose a provoqué sur la toile et dans le milieu du journalisme culturel ce qu'il est convenu d'appeler un "buzz », le bruit ayant couru que cette nouvelle édition cédait à la nécessité d'adapter aux nouvelles générations, moins pétries d'humanités classiques, un texte réputé difficile, par l'explicitation notamment de ses citations latines (cf. U. ECO, «J'AI PRATIQUÉ... »).

43. D. CHAVARRÍA, L'EIL..., « NOTE DE L'AUTEUR », P. 10.

44. México, Joaquín Mortíz ; Montevideo, Editorial Graffiti ; Barcelona, Planeta.

45. U. ECO, «J'AI PRATIQUÉ... ».

46. Les deux éditions comprennent aussi une dédicace à «Hilda [et] Alejo Carpentier » et, pour l'édition française, des «remerciements" au co-directeur de la collection Rivages/noir qui accueille le roman. Les versions espagnoles proposent un intitulé pour chaque chapitre, non reproduit dans l'édition française.

47. P. GARDNER, F. B. JEVONS, A MANUAL OF GREEK ANTIQUITIES, LONDON, GRIFFIN AND CO, 1898 ; P.-F. FOUCART, DES ASSOCIATIONS RELIGIEUSES CHEZ LES GRECS. THIASES, ÉRANES, ORGÉONS, PARIS, KLINCKSIECK, 1873.

48. D. CHAVARRÍA, L'EIL..., P. 79, 104, 197..., ET PASSIM.

49. L. BROCHE, J.-CHR. SARROT, LE ROMAN POLICIER HISTORIQUE..., P. 173-192 («LES GRANDS THÈMES DU POLAR HISTORIQUE »).

50. À titre d'exemple, on comparera les premières lignes de cette entrée commune aux deux lexiques, français et espagnol : «Laconia. Así como el Atica es el territorio de la ciudad-estado de Atenas, Laconia, también llamada Lacedemonia, lo es de Esparta. En cuanto al adjetivo "lacónico" que sobrevive en lenguas modernas, Platón, en su Protágoras (343b), fue el primero en llamar brachylogia lakoniké al lenjuage conciso... » (Guía...) ; « Laconie. Territoire de Sparte (aussi appelé Lacédémonie) » (éd. française).

51. L. BROCHE, J.-CHR. SARROT, LE ROMAN POLICIER HISTORIQUE..., P. 261-271 («FAIRE PARLER AUX PERSONNAGES LE LANGAGE DE LEUR ÉPOQUE ?»).

52. D. CHAVARRÍA, L'CEIL..., « NOTE DE L'AUTEUR », P. 10.

53. E. WAINTROP, « CHAVARRÍA, LABEL HELLÈNE ».

54. D. CHAVARRÍA, L'CEIL..., P. 406.

55. D. CHAVARRÍA, L'CEIL..., P. 103-105, NOTAMMENT. POUR ILLUSTRATION : «TU FERAIS EN SORTE QUE DANS LES PRIÈRES ET LES HYMNES DE LA SOMME, ON LUI DONNÂT UN NOM PLUS BEAU, OUI, AVEC UN ÓNOMA KALLION. LES PORTES DES RÊVES ? OUI, QUEL MAGNIFIQUE ÓNOMA. ET GRECQUEMENT DIT, HAI PYLAI TÔN HYPNÔN ? COMME CELA 
RÉSONNERAIT BIEN LORSQUE TU LE DIRAIS AVEC TA GROSSE VOIX DURANT LA LITURGIE! TU PARCOURUS AINSI BEAUCOUP DE STADES. TU MARCHAS DES PARASANGES, EN ENTENDANT RÉSONNER HAI PYLAI TÔN HYPNÔN, AVEC DES ÉCHOS DE TIMBALES. ET POURQUOI LE GREC RÉSONNAIT-IL MAINTENANT AUSSI BIEN À TES OREILLES, ALORS QUE QUELQUES MOIS PLUS TÔT, TU DÉTESTAIS LE PARLER ? POURQUOI, HEIN ? [...] TU RÉPÉTAS SOUVENT TA DÉCOUVERTE DE VOYAGE ; TU LA RÉPÉTAS EN LYDIEN, EN PHRYGIEN, EN PAMPHYLIEN, EN CARIEN, EN MYSIEN, EN SCYTHE, EN PERSE, EN ÉGYPTIEN, EN BABYLONIEN; MAIS EN AUCUNE LANGUE DES TERRES HABITÉES ELLE NE SONNAIT AUSSI BIEN QUE HAI PYLAI TÔN HYPNÔN, POPOPOM! ET TU DÉCIDAS À CET INSTANT PRÉCIS QU’À CHAQUE FOIS QUE LE NOM BIEN CADENCÉ DES PORTES DES RÊVES SERAIT PRONONCÉ DURANT LES SERVICES RELIGIEUX, UNE TIMBALE DE CUIVRE SONNERAIT POPOPOM ! OU PLUTÔT, POPOMPE POPOPOM ! [...] ET TU MARCHAIS CONTENT, POPOMPE POPOPOM ! »

56. A. ARTAUD, HÉLIOGABALE, OU L'ANARCHISTE COURONNÉ, PARIS, DENOËL ET STEELE, 1934.

57. U. ECO, APOSTILLE..., P. 62.

58. D. CHAVARRÍA, L'EIL..., P. 117 : «- TOUT EN TOI EST HYPOTHÈSE! LUI AVAIT DIT UN JOUR THÉOPOMPE DE LAMPSAQUE. AU DÉBUT, LE SURNOM L'AVAIT GÊNÉ. IL LUI FAISAIT PENSER À HYPOTHÈQUE. IL AVAIT RI DE SES BEAUX YEUX NOIRS ET S'ÉTAIT PASSÉ UN BRAS SOUS LA NUQUE. - N'APPELONS-NOUS PAS HYPOTHÈSES [...] LES SUPPOSITIONS QUI SERVENT DE BASE AUX THÉORÈMES? [...] N'APPELONS-NOUS PAS HYPOTHÈSE TOUT CE QUI SOUTIENT QUELQUE CHOSE?»

59. D. CHAVARRÍA, L'EIL..., P. 332.

60. D. CHAVARRÍA, L'EIL..., P. 428.

61. D. CHAVARRÍA, L'CEIL..., P. 80.

\section{AUTEUR}

\section{MAGALI SOULATGES}

Université d'Avignon et des Pays de Vaucluse

Institut de Recherche sur la Renaissance, l'Âge classique

et les Lumières (UMR 5186) - Université de Montpellier 3

magali.soulatges@univ-avignon.fr 